\title{
Hot Shocked Gas Along the Helical Jets of NGC 4258
}

\author{
G. Cecil
}

Dept. Physics \& Astronomy, U. North Carolina, Chapel Hill, NC 27599, $U S A$

\begin{abstract}
I summarize the results of our multifrequency study of the helically twisted jets in the Seyfert galaxy NGC 4258. [N II], H $\alpha$, and [O III] Fabry-Perot datacubes obtained with the HIFI system on the U. Hawaii $2.2 \mathrm{~m}$ telescope form the basis of this work. ROSAT $\mathrm{x}$-ray multiband images (HRI \& PSPC), CO spectra, and unpublished VLA 6 and $20 \mathrm{~cm}$ maps are correlated with these spectral grids. Together these data reveal clear signatures of high-velocity $\left(>300 \mathrm{~km} \mathrm{~s}^{-1}\right)$ shocks along the jets that suggest efficient entrainment of ambient molecular gas.
\end{abstract}

\section{Introduction}

The jets were furst discovered by Profs. Courtès and Cruvellier in their pioneering survey of galaxies using sensitive focal reducers (1961). These fascinating objects have been extensively studied in the radio continuum (e.g. van der Kruit et al 1972) and visual emission lines (e.g. Martin et al 1989.) Recent kinematic studies trace their interaction with large complexes of molecular clouds (Krause et al 1990; Plante et al 1991), and support the notion that they continue to flow in the dense gas disk. I note in passing an intriguing aspect of the SE jet, namely its organization both spatially (Ford et al 1986) and kinematically (Cecil, Wilson \& Tully 1992, CWT hereafter) into distinct, helically twisted strands when observed at $<3^{\prime \prime}$ resolution (so far attained only at visual wavelengths).

Active galaxies emit over a very large frequency range. One process of particular interest - entrainment of the galaxy ISM by a jet - should proceed at temperatures within the range of $x$-ray telescopes. Indeed, CWT used the Einstein HRI image (Fabbiano, Kim, \& Trinchieri 1992) to show that the SE jet is a resolved $\mathrm{x}$-ray source, with $\mathrm{L}_{\mathrm{x}} \approx 2 \times 10^{40} \mathrm{ergs}^{-1}$. The tighter point-response of the ROSAT HRI has shown us, in a $27 \mathrm{ksec}$ exposure (Cecil, Wilson, \& Depree 1994), that the jets emit most of the soft x-rays in this galaxy. Figure I summarizes the multi-frequency correlations. Their comparative nearness, complex spatio-kinematic structure, strong line flux, and large extent $\left(900 \mathrm{~km} \mathrm{~s}^{-1}\right.$ and $5^{\prime}=10 \mathrm{kpc}$ ) make these jets ideal targets for imaging spectrophotometers.

\section{X-ray Continuum \& Visual Emission-Line Fluxes}

We used our HRI image as a mask to extract the average $x$-ray spectrum of the jets from the PSPC exposure of NGC 4258 in the ROSAT archive. Whatever the absorption, simple free-free, exponential, and power-law forms could not simul- 
taneously fit all energy bands of the PSPC image. However, a Raymond-Smith plasma with $T=0.3 \mathrm{keV}$ did provide a reasonable fit. We can exclude synchrotron and inverse Compton origins for the $x$-ray emission (see Cecil, Wilson, $\&$ De Pree 1994.) Moreover, the decreasing hardness of the jet $x$-ray spectrum toward larger distance from the nucleus appears to be consistent with the lower temperatures expected from the slower jet speeds inferred from our Fabry-Perot study (CWT). Shocked gas cooling through $3 \times 10^{6} \mathrm{~K}$ can indeed reproduce the observed (CWT) emission line-velocities and gaseous excitation. Thus, we have found the tightest correlation seen to date between thermal $\mathbf{x}$-ray emission and a radio jet. The fit might improve by allowing for temperature changes along the jet, which should be practical with the $3 \times$ longer PSPC exposure recently obtained by Pietsch et al. A successful shock model will need more spatio-kinematic constraints and line fluxes from imaging spectrophotometers.

Using the luminosity derived from our $x$-ray spectral fit, we constrain the average gas density in the brightest part of the jets. These are related by

$$
L(T)=V_{\text {eff }} n_{e x} n_{p x} \Lambda(T) \quad \text { erg } \mathrm{s}^{-1}
$$

with $V_{\text {eff }}$ the gas volume, $n_{e x}\left(n_{p x}\right)$ the electron (proton) density of the hot gas, and $\Lambda(T)$ the cooling function. Near $T_{6}=T / 10^{6} \mathrm{~K}$ the latter is approximated by (McKee 1987)

$$
\Lambda\left(T_{6}\right)=1.6 \times 10^{-22} \sqrt{T_{6}} \text { erg } \mathrm{cm}^{3} \mathrm{~s}^{-1}
$$

Our HRI image shows that the jets have total length $l=3 ! 5(7.4 \mathrm{kpc})$ in x-rays. We assume that the hot gas lies in a cylinder of elliptical crosssection and has extent no larger than the bright emission in this image (15".5 after correcting for the HRI point-response.) This upper limit of $540 \mathrm{pc}$ on the FWHM of the jet implies (for disk inclination $72^{\circ}$, e.g. CWT) that the $x$-ray gas lies within $V_{\mathrm{c}}=\pi l(a b) / 4=1.7 \times 10^{65} \mathrm{~cm}^{3}$, and has effective emitting volume $V_{\mathrm{e}}=L(0.30 \mathrm{kev}) / \Lambda n_{e x} n_{p x}=7.7 \times 10^{61}\left(1 \mathrm{~cm}^{-3} / n_{e x}\right)^{2} \mathrm{~cm}^{3}$ (using the observed HRI jet luminosity $6.5 \times 10^{39} \mathrm{erg} \mathrm{s}^{-1}$.) The filling factor of the hot gas is $\eta_{\mathrm{x}}=V_{\mathrm{x}} / V_{c}$, and its mean density is $n_{e x}=0.02 / \beta \mathrm{cm}^{-3}$, where $\beta=\sqrt{\eta_{\mathrm{x}} V_{\mathrm{c} 65.2}}$ and $V_{c 65.2}=V_{c} / 1.7 \times 10^{65}$. This is $3 \times$ the density found by Pietsch et al. (1994) for the more extensive soft $x$-ray halo of NGC 4258, using the same PSPC exposure. The cooling time in collisional equilibrium is then $\tau_{\mathrm{x}}=4 \times 10^{6} \beta \mathrm{yr}$, less than the $50 \mathrm{Myr}$ cooling time of the synchrotron jets (Martin et al 1989.) The mass of hot gas is $M_{\mathrm{x}}=V_{\mathrm{x}} n m_{\mathrm{H}}=3 \times 10^{6} \beta M_{\odot}=6 \times 10^{4}\left(1 \mathrm{~cm}^{-3} / n_{e x}\right) M_{\odot}$, with $\approx 60 \%$ associated with the $S E$ jet.

From CWT most of the Ha-emitting gas in the SE jet is distributed into three intertwined strands, with total volume $V_{8}=1.2 \times 10^{63} \mathrm{~cm}^{3}$ which is $\approx 1 \%$ that of the cylinder of hot gas. We found (CWT) that the total $\mathrm{H} \alpha$ luminosity of NGC 4258 (uncorrected for reddening) is $7.6 \times 10^{39} \mathrm{ergs} \mathrm{s}^{-1}$, of which $14 \%$ originates from the active jets and $\approx 8 \%=6.1 \times 10^{38} \mathrm{erg} \mathrm{s}^{-1}$ comes from the $\mathrm{SE}$ jet. For case-B recombination, the active emitting volume is $V_{e}=L(H \alpha) / j(H \alpha)=2.8 \times 10^{24} L(H \alpha) / n_{e o}^{2}=1.7 \times 10^{63}\left(1 \mathrm{~cm}^{-3} / n_{e o}\right)^{2} \mathrm{~cm}^{3}$ from the $\mathrm{SE}$ jet. If the $\mathrm{H} \alpha$ gas within the strands has filling factor $\eta_{\mathrm{opt}}=V_{e} / V_{s}$, then $n_{e o} \approx 1.2 / \sqrt{\eta_{\mathrm{opt}}} \mathrm{cm}^{-3}$. The total ionized mass in the SE jet is $M_{\mathrm{opt}}=$ $2.2 \times 10^{-33} L(H \alpha) / n_{e o} M_{\odot}=1.3 \times 10^{6}\left(1 \mathrm{~cm}^{-3} / n_{e o}\right) M_{\odot}$. The average pressures 


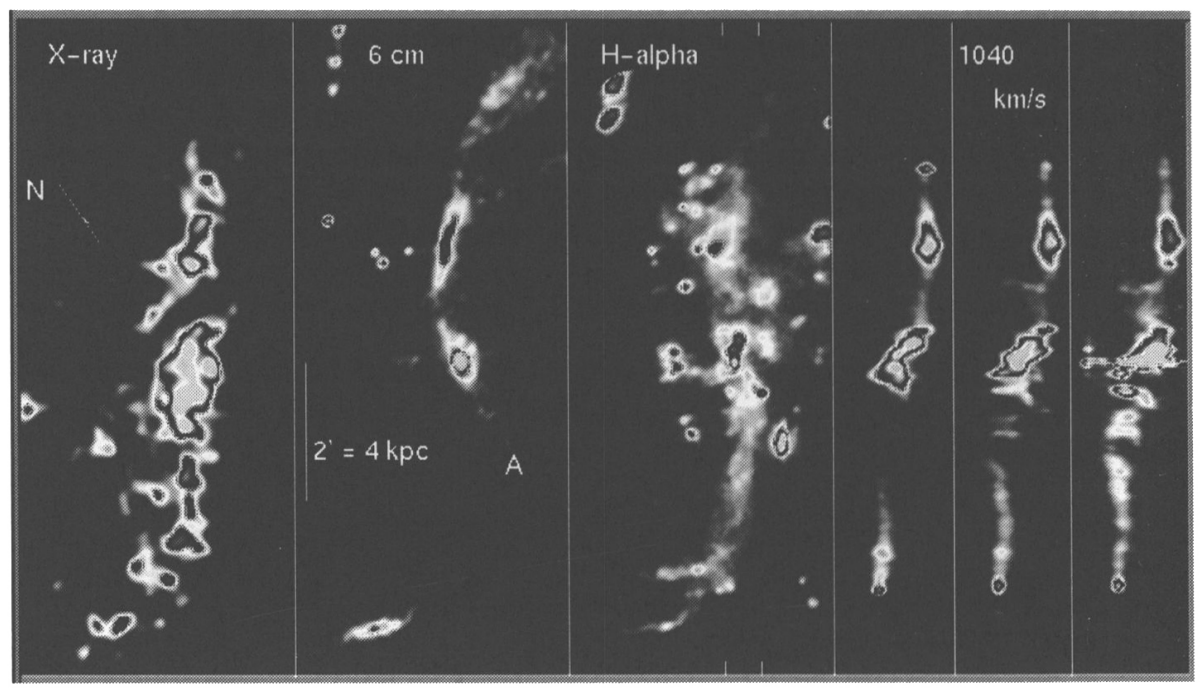

FIGURE I Multifrequency jet structure, and its kinematic manifestations. The galaxy major axis runs top to bottom, north is toward top left, and the nucleus is midway down each plot. The greyscale wraps from white through black in the brightest regions. Left 3 images: (left) $27 \mathrm{ksec}$ exposure with ROSAT HRI ( $8^{\prime \prime}$ FWHM resolution); (middle) unpublished $\lambda 6 \mathrm{~cm}$ continuum image from VLA archive ( $3^{\prime \prime} \mathrm{FWHM}$ ); (right) $\mathrm{H} \alpha$ flux image (2.5 FWHM) made by summing our Fabry-Perot images (CWT) that best show the jets. The SE jet is prominent in x-rays and $\mathrm{H} \alpha$, but is faint at 6 $\mathrm{cm}$ because of its rapidly falling radio spectrum (Cecil, Wilson, \& De Pree 1994). Right 3 images: space-velocity plots made by slicing through the $\mathrm{H} \alpha$ Fabry-Perot datacube of CWT (resolution $70 \mathrm{~km} \mathrm{~s}^{-1}$ ) across the interval shown by ticks in the $\mathrm{H} \alpha$ image. Each slice spans $1040 \mathrm{~km} \mathrm{~s}^{-1}$. Note the $\pm 350 \mathrm{~km} \mathrm{~s}^{-1}$ oscillations in peak velocity just below (SE of) the nucleus. 
of hot gas, $\mathrm{H} \alpha$ gas, and relativistic electrons (derived by Hummel, Krause, \& Lesch [1989; HKL hereafter] assuming the electrons are in energy equipartition at larger radii along the jet) in the $\mathrm{SE}$ jet are $\left(9.6 / \beta, 1.7 / \sqrt{\eta_{\mathrm{opt}}}\right.$, and 30$) \times 10^{-12}$ dynes $\mathrm{cm}^{-2}$, respectively. So, the pressures may be similar.

The mass of both visual line and x-ray-emitting gas can be written as $X\left(1 \mathrm{~cm}^{-3} / n_{e}\right) M_{\odot}$ because the emissivity in both cases is $\propto n_{p} n_{e}$. If we assume that the two gases are in pressure equilibrium, we find the relative values of $n_{e}$ $\left(n_{e o} / n_{e x}=350\right)$, mass $\left(M_{\mathrm{opt}} / M_{\mathrm{x}}=0.06\right)$, and filling factor $\left(\eta_{\mathrm{opt}} / \eta_{\mathrm{x}}=V_{c 65.2} / 32\right)$ for the two gases. If we further assume that $\eta_{\mathrm{x}} \approx 1$ (i.e. the x-ray emitting gas fully fills the cylinder) then the last relation implies $\eta_{\text {opt }}=0.03$ if $V_{\mathrm{c} 65.2}=1$.

\section{Correlations with emission-line kinematics}

The right-hand panels in Figure I show that detailed changes in the kinematics of the jets are correlated with changes in the radio and $\mathrm{x}$-ray morphologies. One sees that within $35^{\prime \prime} \mathrm{SE}$ of the nucleus the jet velocities oscillate by \pm 300 $\mathrm{km} \mathrm{s}^{-1}$ around the mean motions of the bar-forced gas disk (CWT). Such speeds are almost adequate to shock gas to a peak temperature $0.3 \mathrm{keV}\left(T_{s}=3.2 \times\right.$ $10^{5} V_{\mathrm{B} 7}^{2} / x_{\mathrm{ts}} \mathrm{K}$, where $V_{B 7}$ is the shock speed in units of $100 \mathrm{~km} \mathrm{~s}^{-1}$ and $x_{t 8}$ is the particle concentration relative to protons $=2.3$ for a fully ionized preshock medium with $10 \%$ He abundance [Hollenbach \& McKee 1979].) These authors show that $T_{g}$ is not influenced by magnetic fields if the Alfvén Mach number $v_{s} / v_{A}$ exceeds 6 . CWT estimated that $v_{A} \lesssim 50(B / 20 \mu G) \mathrm{km} \mathrm{s}^{-1}$ from the radio data of HKL, so the fields do not appear to inhibit compression. Significantly, gas speeds become sub-Alfvénic beyond the possible end of the active jet (" $\mathrm{A}$ " in Fig. I.) The profiles in the bottom-half of Figure I continue their spatio-kinematic wiggles, suggesting a transition from hydrodynamic to MHD instabilities. Sadly, our attempts to study this region with $2 \times$ better spatial and velocity resolutions have been foiled for the past 2 years by bad weather at the CFHT.

\section{Conclusions}

Entrainment is a complex process, and current numerical simulations cannot track the multiphase ISM that is involved, so are probably irrelevant. By combining our measurements of $10^{6} \mathrm{~K}$ gas along the optical and radio jets to kinematic and line-flux evidence from our imaging spectrophotometry, we can build a convincing case for entrainment. And give the modelers something to model.

Acknowledgments. to my collaborators A. Wilson \& C. De Pree, and to NASA and NSF for grants.

\section{Discussion}

Hippelein: Did I understand you correctly that the radio jet and the noodle-like $\mathrm{H} \alpha$ jet don't follow the same direction, and don't interfere with each other?

Cecil: The $3^{\prime \prime} \lambda 6 \mathrm{~cm}$ VLA map shows that the directions of radio and $\mathrm{H} \alpha$ jets coincide beyond $35^{\prime \prime} \mathrm{SE}$ of the nucleus and to the NW. At $<35^{\prime \prime}$ radius the 
active radio jet is displayed $\approx 45^{\circ}$ (deprojected) ahead (in the sense of galactic rotation) of the brightest $\mathrm{H} \alpha$ emission. However, the distribution of ionized gas is complex near the nucleus, the $\mathrm{H} \alpha$ jet is $\approx 12^{\prime \prime}$ across, and there is strong visual extinction along the leading edge of the jet. So, the radio image is likely showing the locus of the active jet near the nucleus. I think that the jet terminates at the bright blob " $A$ " in Figure $I$.

\section{References}

Cecil, G., Wilson, A.S., and De Pree, C.D. 1994, ApJ, submitted Cecil, G., Wilson, A.S., \& Tully, R.B. 1992, ApJ, 390, 365 (CWT)

Courtès, G. \& Cruvellier, P. 1961, Comp. Rend. Acad. Sci. Paris, 253, 218

Ford, H.C. et al 1986, ApJ, 311, L7

Hollenbach, P.J. \& McKee, C.F. 1979, ApJS, 41, 555

Hummel, E., Krause, M., \& Lesch, H. 1989, A\&A, 211, 266 (HKL)

Kim, D. W., Fabbiano, G., \& Trinchieri, G. 1992, ApJ, 393, 134

Krause, M., Cox, P., Garcia-Barretto, J.A., \& Downes, D. 1990, A\&A, 233, L1

Martin, P., Roy, J.-R., Noreau, L., \& Lo, K. Y. 1989, ApJ, 345, 707

McKee, C.F. 1987, in Spectroscopy of Astrophysical Plasmas, eds A. Dalgarno \& D. Layzer, chap. 9 (Cambridge U. Press)

Pietsch, W. et al, 1994, A\&A, in press

Plante, R. et al, 1991, ApJ, 381, 110

van der Kruit, P.C., Oort, J.H., \& Mathewson, D.S. 1972, A\&A, 21, 169 